\title{
Verschobene Kuren haben schmerzliche Folgen
}

Eine Befragung zur Patientenzufriedenheit aus dem Gasteiner Heilstollen ergab, dass fast jeder Zweite im Zeitraum von April 2020 bis Mai 2021 seine geplante Kur ausgelassen hat. Weitere $15 \%$ aller 830 Befragten haben die Kur verschoben.

Als Gründe für das veränderte Kurverhalten gaben die Befragten an: Einschränkungen durch die CoronaSituation und sich keinem weiteren Infektionsrisiko aussetzen zu wollen. Nur etwa $35 \%$ aller Befragten führten ihre Kur wie geplant durch. Das Auslassen der regelmäßigen Heilstollen-Therapien führte bei dem überwiegenden Teil der Patient*innen zu deutlichen Auswirkungen auf den Gesundheitszustand: $54 \%$ hatten mehr Schmerzen als gewöhnlich, $48 \%$ büßten an Wohlbefinden ein, $30 \%$ mussten mehr Medikamente zu sich nehmen und jede*r Fünfte hatte mehr Arztbesuche als mit einer Kur. Immerhin jede*r Zehnte beklagte mehr Krankheitstage als mit Kur. Umgekehrt beobachteten nur $16 \%$ keine relevanten negativen Auswirkungen durch die fehlende Gesundheitsmaßnahme.

In einer weiteren Frage sollten die Patient*innen Auskunft geben, wel- che Beweggründe sie für eine Kur im Gasteiner Heilstollen haben. Knapp $96 \%$ schätzten besonders die gesundheitliche Heilwirkung und $94 \%$ attestierten der Therapie Effektivität. Für jeweils zwei Drittel der Befragten waren auch der Ablauf und die Organisation sowie die Freundlichkeit der Mitarbeiter*innen ein wichtiger Beweggrund. Noch für jede*n Dritten war die Kompetenz des Ärzt*innenteams, die Wirkung der Zusatztherapien sowie die Besonderheit des Ortes von Relevanz.

》) $54 \%$ hatten mehr Schmerzen als gewöhnlich

Seit 1952 wird im Gasteiner Heilstollen das natürliche Klima für eine kombinierte Low-Dose-Radon- und Hyperthermie-Therapie (LDRnHT) vor allem gegen Schmerzen genutzt. Schwerpunktmäßig nutzen Patient*innen mit
Spondyloarthriten und rheumatischen Erkrankungen das im ehemaligen Bergwerks-Stollen natürlich vorkommende Klima zur Kur. Vor PandemieBeginn kamen jährlich etwa 14.000 Patient*innen mit knapp 90.000 Einfahrten zur Kur.

Nähere Informationen unter www. gasteiner-heilstollen.com.

Hinweis des Verlags. Der Verlag bleibt in Hinblick auf geografische Zuordnungen und Gebietsbezeichnungen in veröffentlichten Karten und Institutsadressen neutral.

rheuma plus $2022 \cdot 21: 32$

https://doi.org/10.1007/s12688-02200495-6

(c) The Author(s), under exclusive licence to Springer-Verlag GmbH Austria, ein Teil von Springer Nature 2022

Hier steht eine Anzeige. 